\title{
Only the Strong: Restricting Situation Variables
}

\author{
Ezra Keshet \\ MIT
}

\section{Introduction}

Linguists often assume that possible worlds and times are represented as pronouns in natural language (see Cresswell 1990, Percus 2000, Kusumoto 2005, Keshet 2008). This paper will construe such pronouns as world-time pairs, which will be referred to as situations, for simplicity. A predicate taking such a pair as an argument is evaluated in the world and the time specified by that situation, effectively determining whether it is de re or de dicto. Researchers such as Percus (2000) have noted that such a system overgenerates and proposed generalizations describing where this overgeneralization occurs. This paper will examine three such generalizations: Percus's (2000) Generalization X, a generalization by Musan (1997), and a new generalization covering modifiers of nouns. Ultimately, a unified generalization will be proposed, stating that only strong DPs ever may receive a de re interpretation. An explanation for this generalization is offered, involving a change in the semantic type system, under which only strong determiners may take situation pronouns.

\section{Restrictions on Situation Pronouns}

The first question to arise once situation pronouns are posited is whether there are any constraints on these pronouns' distribution and indexing. To answer this question, I will begin by assuming the least restrictive theory possible concerning the distribution and indexing of situation pronouns. This null hypothesis might be as follows:

(1) Free Situation Pronoun Hypothesis: A situation pronoun may be freely inserted and indexed wherever it is sister to a node of type $\langle s, \alpha\rangle$.

This section describes three generalizations (one new) pertaining to cases where the Free Situation Pronoun Hypothesis overgenerates. Last, a new, unified generalization is proposed to cover all three cases.

Thanks to Danny Fox, Irene Heim, Alan Bale, Jon Gajewski, Yael Sharvit, Jon Nissenbaum, Junri Shimada, and Yasutada Sudo. 


\subsection{Generalization $X$}

Percus (2000) proposes his Generalization X based on the fact that the following sentence is missing a reading predicted under the Free Situation Pronoun Hypothesis:

(2) Mary thinks my brother is Canadian. (= Percus's 26a)

The embedded sentence in (2) has two predicates which take type-s arguments - my brother and is Canadian - and therefore, according to the Free Situation Pronoun Hypothesis, a structure like (4) should be available, given the definitions in (3).

a. $\quad$ my brother $\rrbracket=\lambda s_{s} . \lambda x_{e} . x$ is my brother in $s$

b. $\quad$ is Canadian $\rrbracket=\lambda s_{s} \cdot \lambda x_{e} \cdot x$ is Canadian in $s$

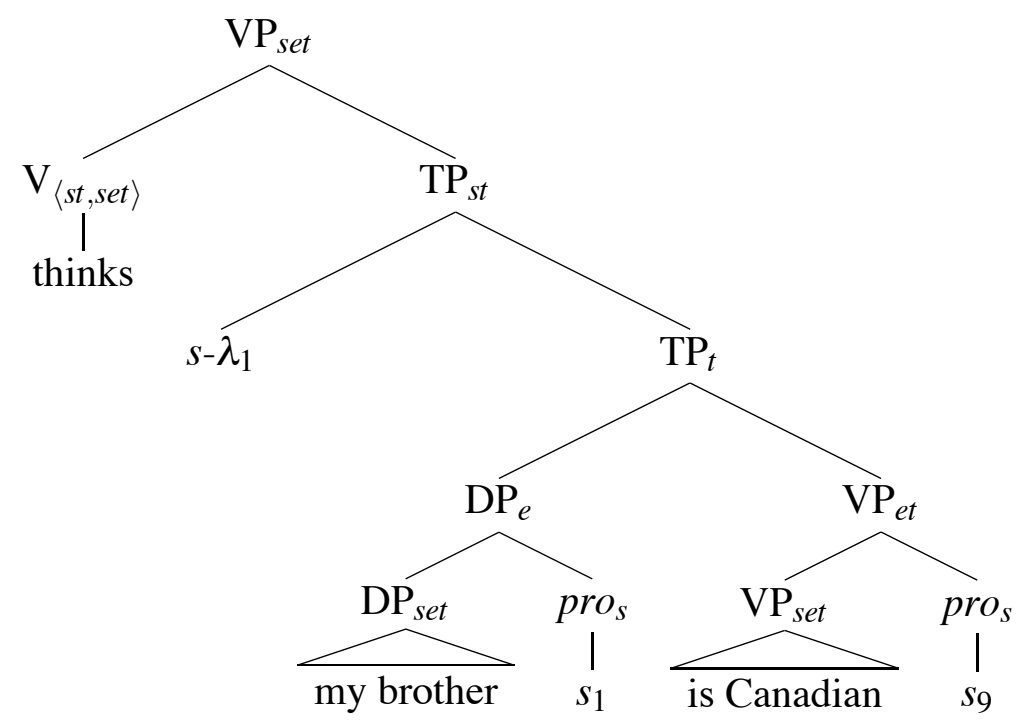

When a situation pronoun $s$ in the scope of an intensional operator $\alpha$ is not bound by a $\lambda$ operator directly below $\alpha$, any predicates that are evaluated in the world and time denoted by $s$ can be de re (with respect to $\alpha$ ). In (4), therefore, the VP is Canadian can be de re, since it is evaluated at the world and time determined by the situation pronoun $s_{9}$, which is not bound by the $\lambda$ operator directly below the verb thinks. Note that in order for this structure to be non-trivial, the subject my brother must be de dicto. Otherwise, there would be nothing at all bound by the $s-\lambda_{1} .{ }^{1}$

Percus notes that despite this possible structure, there is no reading where is Canadian is de re. He describes the meaning of such a reading as follows:

(5) “... we would take the sentence to be true whenever there is some actual Canadian who Mary thinks is my brother - even when this person is not my brother in actuallity, and even when Mary mistakenly thinks that he is not Canadian" (p. 200).

\footnotetext{
${ }^{1}$ See Percus's footnote 18, p. 200, for discussion.
} 
In other words, it would mean that someone Mary thinks is my brother is Canadian. Based on this and other evidence, Percus proposes his Generalization X:

(6) Generalization $\mathbf{X}$ : The situation pronoun that a verb selects for must be coindexed with the nearest $\lambda$ above it $(=34$, p. 201).

\subsection{Musan's Generalization}

Musan (1997) makes the observation that while strong DPs can be evaluated at a time independent from the main predicate of their clause, weak NPs must be evaluated at the same time as this main predicate:

(7) Musan's Generalization: A noun phrase can be temporally independent if and only if it is in a strong DP $(\approx$ Musan's 10, p. 60$){ }^{2}$

(8) Definitions: A noun phrase is temporally dependent if its time of evaluation must be same as the time of evaluation for the main predicate of its sentence. Otherwise, the noun phrase is temporally independent.

Take the following sentence, for instance, which is an adaptation of Musan's examples:

(9) Some members of congress knew each other in college. In fact, ...

a. ... three U.S. Senators were attending Harvard together in 1964.

b. \#... there were three U.S. Senators attending Harvard together in 1964.

In (9-a), the subject three U.S. Senators may be evaluated in the present, meaning something like three current U.S. Senators. The VP were attending Harvard together, on the other hand, is evaluated in the year 1964. If the two were instead evaluated at the same time, the sentence would sound odd, since most college students are too young to be senators (who must be at least 30 years old according to the U.S. constitution). And, in fact, (9-b) does sound odd for this very reason: the two contradictory descriptions are required to hold at the same time. According to Musan, this odd reading is due to the fact that three U.S. Senators is a weak NP in (9-b), as evidenced by the fact that it appears in the Existential There Construction. Since it is weak, the NP must be evaluated at the same time as its main predicate, attending Harvard together.

I assume that the NP and the post-nominal predicate in an Existential There Construction are interpreted via the Predicate Modification composition rule. Discounting situation arguments for a moment, I will assume a structure similar to the one shown in (10):

(10) a. There are students in the room.

\footnotetext{
${ }^{2}$ Musan later revises this generalization to include facts about existence-independent predicates like is famous; I will ignore such predicates.
} 
b.

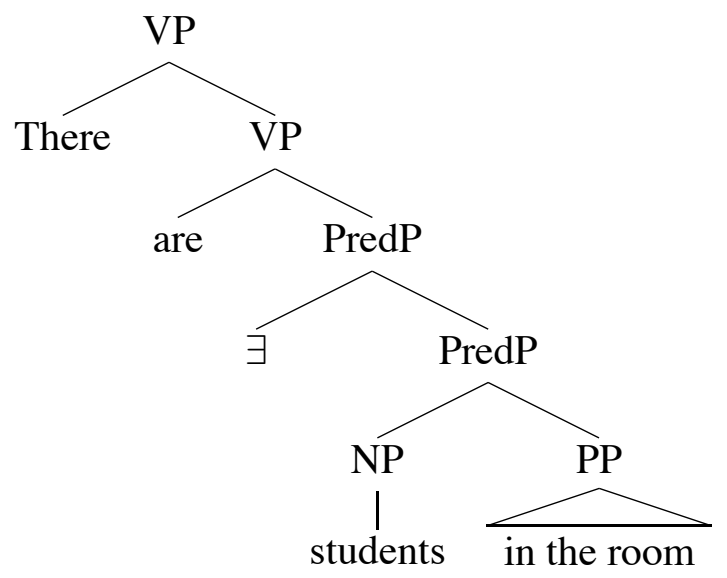

Here the NP students combines with in the room via Predicate Modification to form a node denoting students who are in the room, before undergoing existential closure. Once you add situation pronouns into the picture, the Free Situation Pronoun Hypothesis predicts at least the three structures in (12) for the node marked PredP in (10), assuming the lexical entries in (11):

(11) a. $\quad$ students $\rrbracket=\lambda s . \lambda x_{e} \cdot x$ comprises students in $s$

b. $\quad$ in the room $\rrbracket=\lambda s . \lambda x_{e} . x$ is in the room in $s$

a.

b.

c.
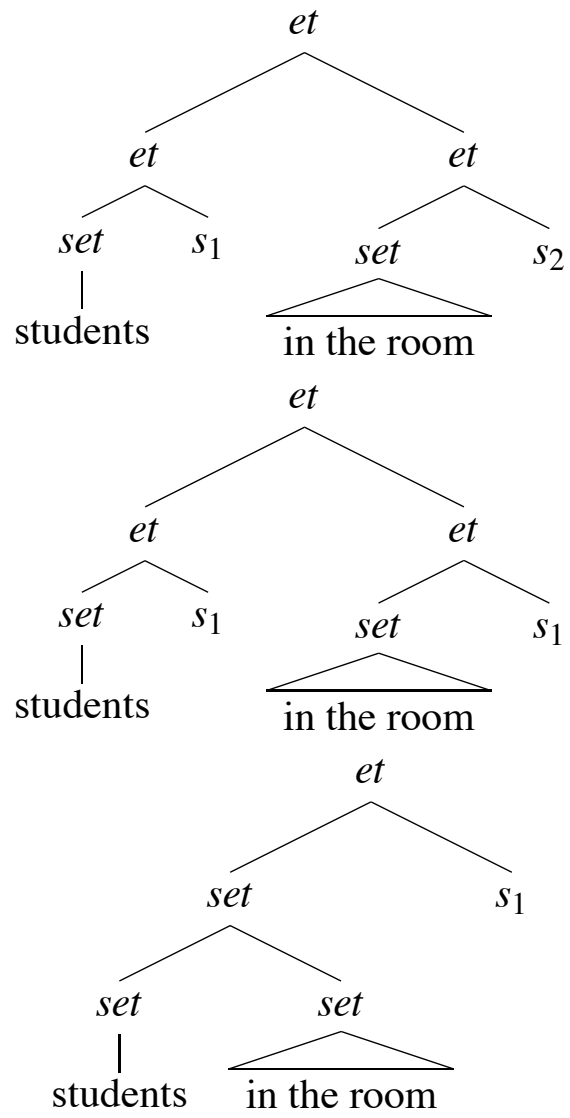

In each structure above, students and in the room combine via Predicate Modification. (This combined predicate is then existentially closed higher in the structure.) 
However, how these two phrases combine with situation pronouns differs in each structure. In (12-a), the NP and the predicate take two different situation variables, $s_{1}$ and $s_{2}$; in (12-b), they take two coindexed pronouns; and in (12-c), they only take one pronoun. Musan's Generalization is only compatible with the latter two structures, where the NP three students is evaluated at the same world and time as the predicate in this room.

\subsection{Nouns and Intersective Modifiers}

The new generalization arises in another case of two phrases being composed via Predicate Modification: a noun and an intersective modifier (Jackendoff 1977). In an extensional system, such a configuration looks like the following:

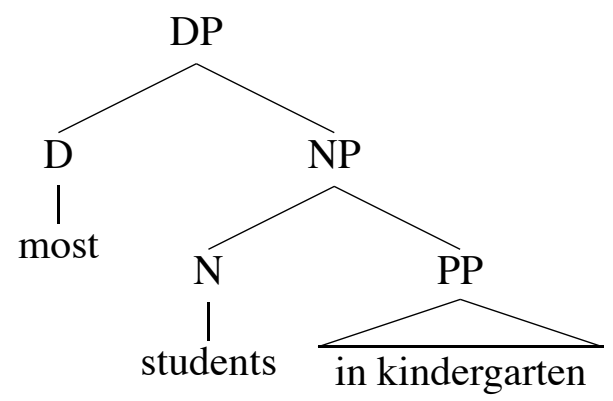

The noun and the modifier in (13) combine via Predicate Modification. Once situation pronouns are factored in, the Free Situation Pronoun Hypothesis would predict all the structures in (15) for (13), given the lexical entries in (14):

a. $\llbracket$ students $\rrbracket=\lambda s_{s} . \lambda x_{e} \cdot x$ comprises students in $s$

b. $\quad$ in kindergarten $\rrbracket \lambda s_{s} . \lambda x_{e} . x$ is in kindergarten in $s$

c. $\quad \llbracket \mathbf{m o s t} \rrbracket=\lambda P_{e t} . \lambda Q_{s e t} . \lambda s_{s}$. for most $x$ such that $P(x) \cdot Q(s)(x)^{3}$

a.

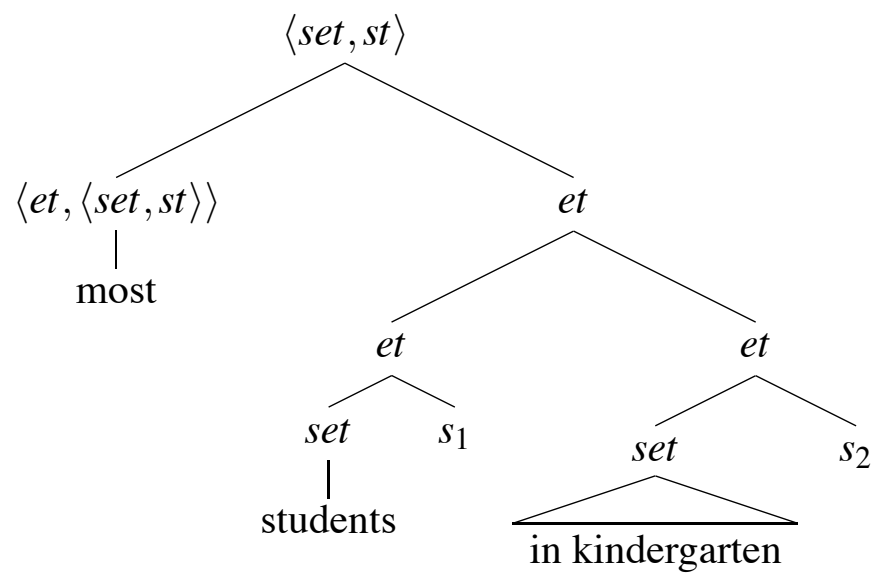

\footnotetext{
${ }^{3}$ See section 4.2 for a revised definition for generalized quantifiers.
} 
b.

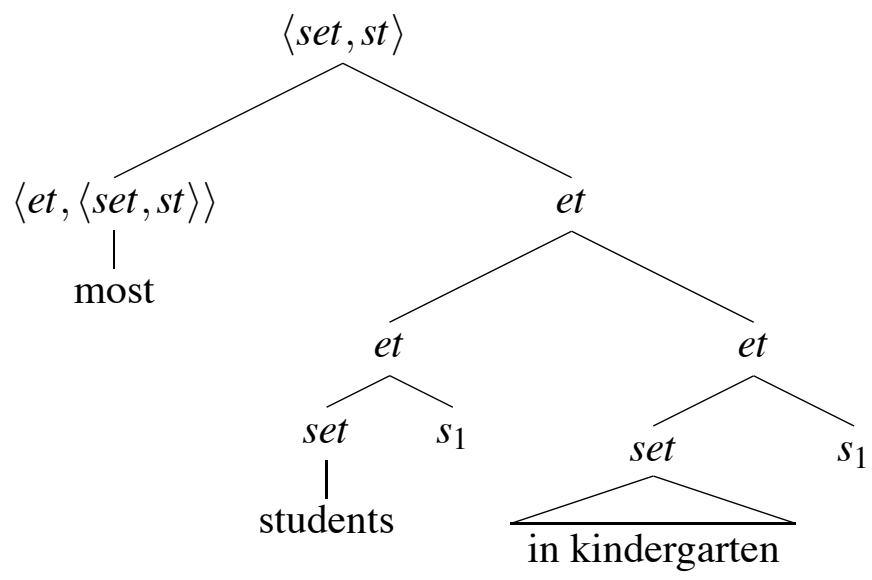

c.

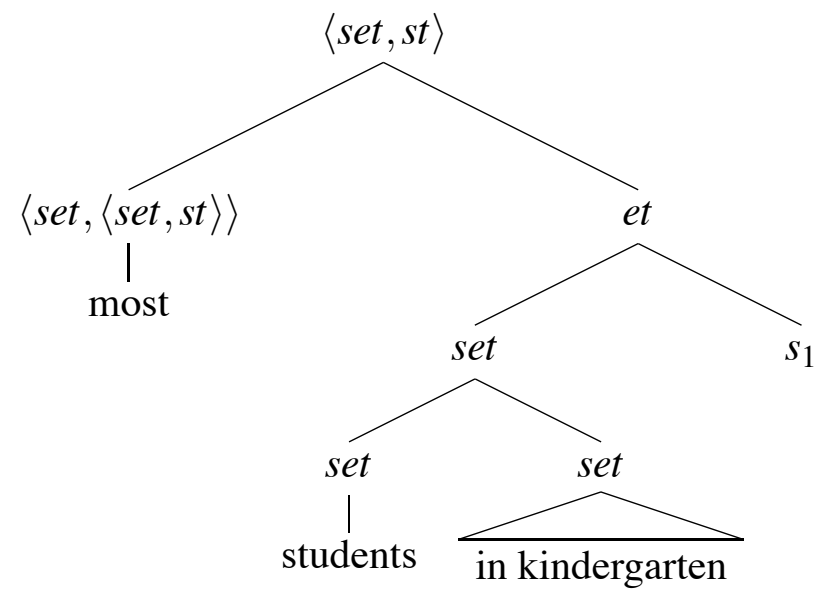

In (15-a), the noun and its modifier take different situation pronouns; in (15-b) they take coindexed pronouns; and in (15-c) they only take one situation pronoun. The generalization defended in this section (given in (16)) is upheld only in the latter two structures, where the noun and its modifier must be interpreted at the same world and time.

Noun-Modifier Generalization ${ }^{4}$ : A noun and an intersective modifier must be evaluated at the same time and world.

I will show evidence for this generalization, first relative to times and then relative to possible worlds:

\section{\#In 1964, every U.S. Senator at Harvard got straight A's.}

$$
\text { Every U.S. Senator who was at Harvard in } 1964 \text { got straight A's in college. }
$$

If the noun U.S. Senator in (17) and its modifier at Harvard could hold at different times, then the sentence might mean the same as $(18)^{5}$. However, consistent with the Noun-Modifier Generalization, this reading is not available. The sentence sounds odd since it entails that there were people who were sitting senators and at

\footnotetext{
${ }^{4}$ A suggestion along these lines was first made to me by Jon Gajewski, p.c.

${ }^{5}$ In (18), the noun holds at the same time as the entire modifier who was at Harvard in 1964; the phrase at Harvard holds at the time shifted backwards by the past tense on was.
} 
Harvard at the same time.

(19) \#Mary thinks the married bachelor is confused.

The reasoning follows similarly for the case in (19). Here, bachelor and married must be in the same world, despite the fact that it leads to an odd reading.

\subsection{Unified Generalization}

Consider the following summary of the results above:

\begin{tabular}{llll} 
Phrase & Domain & Non-local? & Generalization \\
\hline Strong DP & CP & $\checkmark$ & \\
Weak NP & CP & X & Musan \\
VP & CP & X & Percus (X) \\
Noun Modifier & NP/DP & X & Noun-Modifier
\end{tabular}

Musan's Generalization states that weak NPs must be de dicto within the CP, Percus's Generalization $\mathrm{X}$ that VPs must be de dicto within the $\mathrm{CP}$, and the NounModifier Generalization that modifiers of nouns must be "de dicto" in the sense that they are evaluated at the time and world local to their head noun. The only items that are ever de re are strong DPs. One way of combining these three generalizations is the following:

(21) A node may be interpreted de re (i.e., at a non-local world or time) iff it is a strong DP.

\section{Observation}

Before an explanation of this unified generalization is tendered, first let us examine a pattern to the structures which violate the generalization. Repeated below are structures representative of violations of Percus's Generalization X, Musan's Generalization, and the Noun-Modifier Generalization. In each case, a situation pronoun combines with a node to form another node whose denotation has an extensional type - i.e., a type containing no situations ( $s$ 's). These nodes have been framed by boxes in the diagrams below: 
(22) Violation of Percus's Generalization
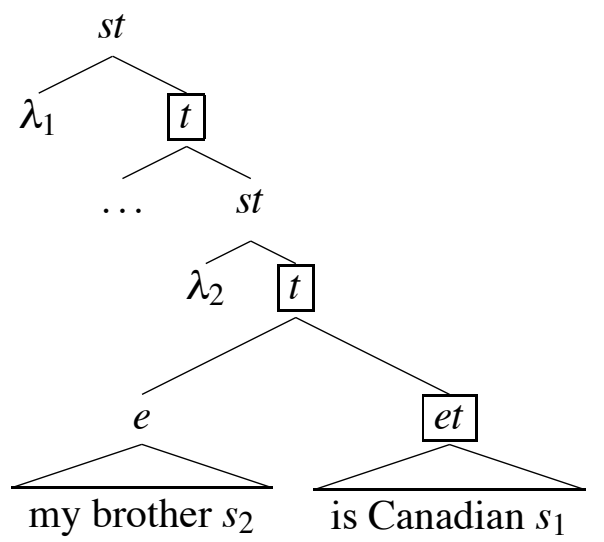

(23) Violation of Musan's Generalization
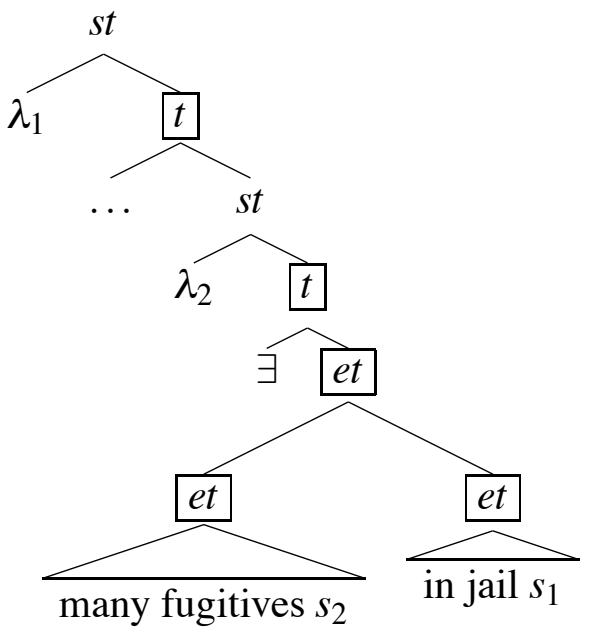

(24) Noun-Modifier Generalization Violation

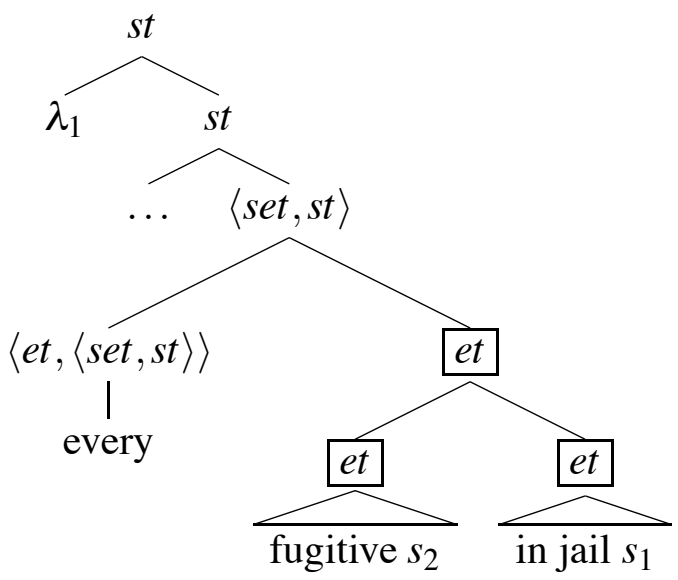

Based on this observation, the intuition behind the proposal below will be the following: if there were no extensional types, structures such as these would not be possible, preventing violations of the generalizations. 


\section{Proposal}

The implementation of this intuition comes in the form of a modification to the semantic type system, along the lines of that proposed by Cresswell (1973) and Kratzer (1991). The new system is as follows (cf. Heim and Kratzer 1998):

\section{Semantic types}

a. $\quad e, s$, and $p$ are types.

b. If $\alpha$ and $\beta$ are types, then $\langle\alpha, \beta\rangle$ is a type.

c. Nothing else is a type.

(26) Semantic domains

Let $W$ be the set of all possible worlds. Let $I$ be the (ordered) set of all times. Let $S$ be $W \times I$, the set of all situations (i.e., pairs of worlds and times). Associated with each situation $s$ in $S$ is the domain of all individuals existing in $s$. Let $D$ be the union of the domains of all situations.

a. $D_{e}=D$

b. $D_{s}=S$

c. $D_{p}=$ the power set (set of all subsets) of $S$

d. If $\alpha$ and $\beta$ are semantic types, then $D_{\langle\alpha, \beta\rangle}$ is the set of all functions from $D_{\alpha}$ to $D_{\beta}$.

The major difference between this system and the one in Heim and Kratzer (1998) is that this system has no type $t$ (for truth value). Instead there is a type $p$ (for proposition) which is the type for sets of situations. (This type is roughly equivalent to $\langle s, t\rangle$ in Heim and Kratzer's system.) A simple sentence, such as it's raining, therefore will denote a set of situations, and the utterance of such a sentence will assert that the situation comprising the real world and the utterance time is in that set.

\subsection{Explanation of Generalizations}

Let us see how the examples which were problematic for the Free Situation Pronoun Hypothesis are treated in this new system. First, consider our example for Percus's Generalization X:

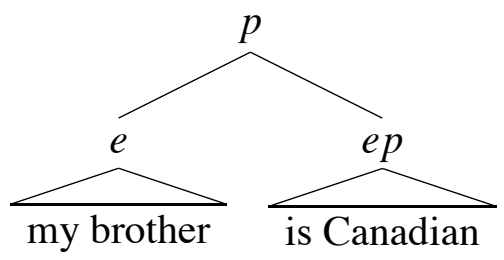

Whereas in the old system there was a question of whether one-place predicates had the type $\langle e,\langle s, t\rangle\rangle$ or $\langle s,\langle e, t\rangle\rangle$, under the new system all such predicates are of type $\langle e, p\rangle$. Therefore, in (27), the VP does not take an argument of type $s$ at all, so it can only receive a de dicto interpretation. 
Next, consider the example for Musan's Generalization:

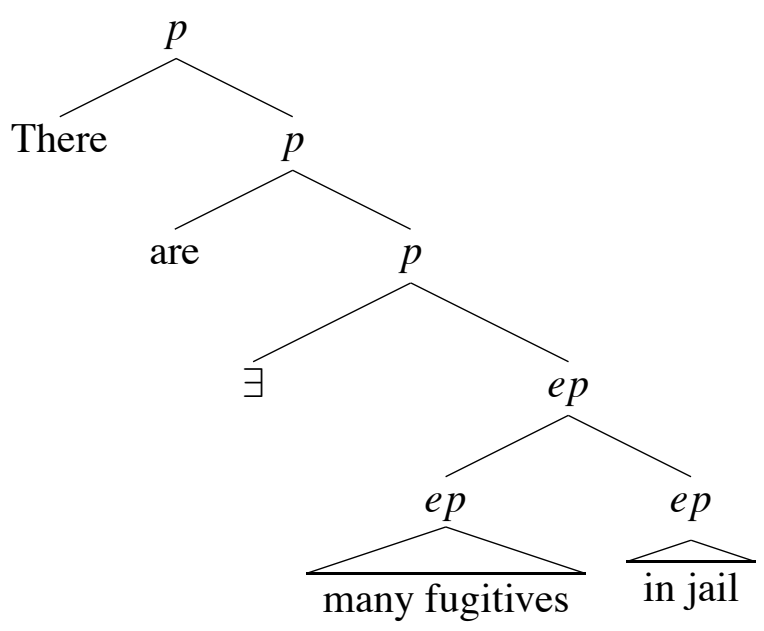

Weak DPs in this new system are also of type ep. In the Existential There Constructure, they combine with their main predicates via Predicate Modification before existential closure applies (Milsark 1974, Heim 1982, Diesing 1992). Once again, there is no type-s argument.

Last, (29) is an example of the Noun-Modifiers Generalization:

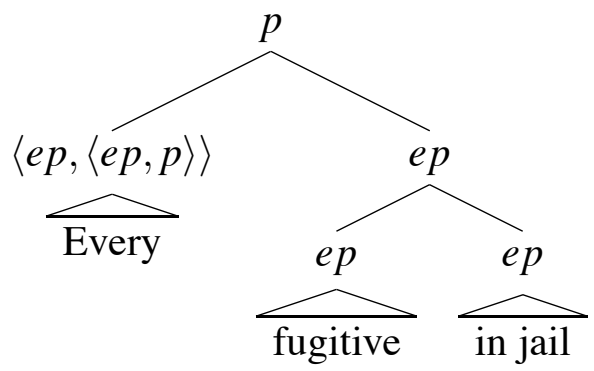

And similarly to the previous case, a noun and its modifiers combine via Predicate Modification, without a type-s argument.

\subsection{Strong Quantifiers}

So far, the new system has achieved the goal of ruling out cases in which a node that is not a strong DP receives a de re interpretation. Now let us turn our attention to strong DPs themselves and see how they can receive de re interpretations. Strong determiners, I assume, take a situation argument as represented below (the $\beta$ operator will be explained in a moment): 
(30)

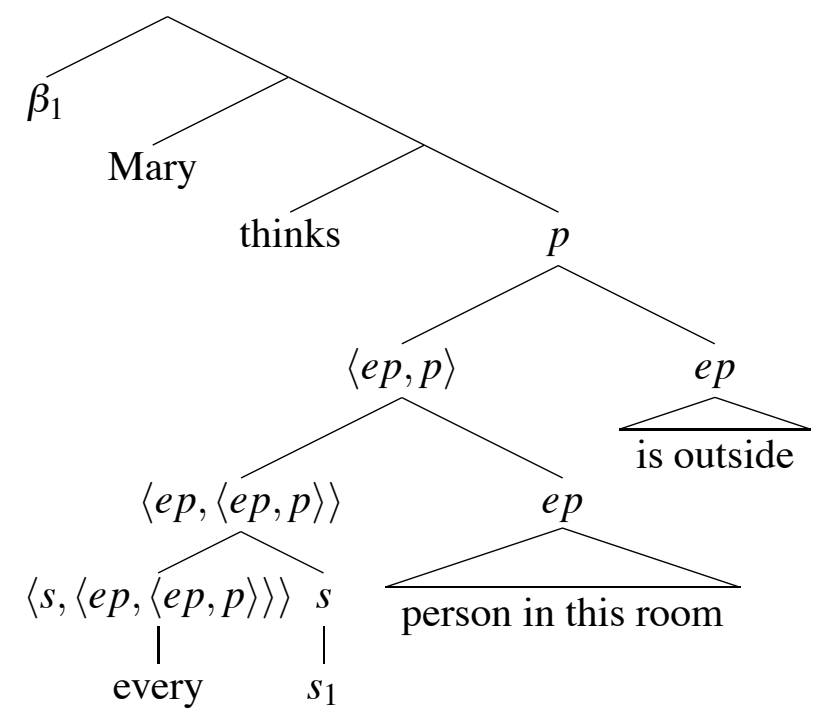

In (30), the determiner every has the type $\langle s,\langle e p,\langle e p, p\rangle\rangle\rangle$, and therefore its first argument is a situation pronoun. I further assume that this situation argument determines the time and world in which the quantifier's restrictive clause is interpreted, but not the time and world in which the nuclear scope is interpreted. In this way, the strong DP (i.e., the restrictive clause) may be de re, but the VP (i.e., the nuclear scope) may not.

In order to correctly define such a determiner, though, I must first introduce two new operators, whose purpose is to convert objects of type $p$ - sets of situations - into the characteristic functions of those sets, and vice versa. These operators are written $\cup$ and $\cap$ after Chierchia (1998):

$$
\begin{array}{ll}
\text { a. } & \cup P \leftrightarrow[\lambda s . s \in P], \text { for any } P \in D_{p} . \\
\text { b. } \quad \cap f \leftrightarrow\{s: f(s)=1\}, \text { for any } f \in\left[D_{s} \rightarrow D_{t}\right] .
\end{array}
$$

$\cup$ converts a set of situations into a function from situations to truth values and $\cap$ does the reverse. I assume that these operators are only available in the metalanguage, and are tied to the definitions of only a small number of lexical items.

Given these operators, we can now define every:

$$
\llbracket \text { every } \rrbracket=\lambda s \cdot \lambda P_{e p} \cdot \lambda Q_{e p} \cdot \cap\left[\lambda s^{\prime} \cdot \forall x \cdot[\cup P(x)](s)=1 \rightarrow\left[{ }^{\cup} Q(x)\right]\left(s^{\prime}\right)=1\right]
$$

Under this definition, every first takes a situation argument $s$, then two predicates $P$ and $Q$ of type $\langle e, p\rangle$. The situation $s$ is applied to $P$, the quantifier's restrictive clause, via use of the ${ }^{\cup}$ operator. Through use of both the $\cup$ and $\cap$ operators, the denotation of the node containing every once it takes all its arguments is of type $p$ - a set of situations. Notably, this set is linked to the argument of $Q$, the nuclear scope of the quantifier, and therefore the nuclear scope (the VP) will never be de re, only de dicto. Only the restrictive clause, which is linked to the situation argument $s$ may be de re. Therefore, only the strong DP itself may be de re, and not the VP, or any part of the DP. Weak noun phrases do not take such a situation argument and therefore may not be de re, as shown above. 


\subsubsection{Binding}

One remaining question about this system is how the situation pronoun argument of a strong determiner is bound. For this question, we turn to a proposal of Schueler (2007), who adapts the $\beta$ operator of Büring (2005). Büring's $\beta_{i}$ (binder prefix) operator takes a predicate with an open individual argument slot, and binds anything indexed $i$ in its scope to this open argument slot. So, it takes a node of type $\langle e, t\rangle$ and returns one of type $\langle e, t\rangle$; the only change it makes is to the assignment function. Schueler extends the assignment function to return items not only of type $e$, but also of type $s$; he then extends the $\beta$ operator to take nodes with an open situation argument (type $\langle s, t\rangle$ ) and bind co-indexed situation pronouns to this slot. My definition, given in (33), follows Schueler; the only difference comes about due to the new type system and the ${ }^{\cup}$ and $\cap$ operators:

$$
\llbracket \beta_{i} \mathbf{X} \rrbracket^{g}=\cap\left[\lambda s . \cup \llbracket \mathbf{X} \rrbracket^{g[i \rightarrow s]}(s)\right]
$$

Given this operator, the binding works as follows:
a. de re:
[ $\beta_{1}$ Mary thinks [ $\beta_{2}$ every $s_{1}$ boy is outside]].
b. de dicto:
[ $\beta_{1}$ Mary thinks [ $\beta_{2}$ every $s_{2}$ boy is outside]].

When a situation pronoun $s$ is bound by a non-local $\beta$ operator, any nodes whose world and time of evaluation are determined by $s$ will be de re. When a situation pronoun is bound by a local $\beta$ operator, any such nodes will be de dicto.

\section{Conclusion}

This paper has shown how three generalizations constraining the situation at which natural language expressions may be evaluated can be unified into one: namely, that only strong DPs may be evaluated de re. Any method of restricting the system such that only strong DPs can take situation pronouns will suffice to explain this generalization: even simply stipulating it. However, this paper tried to explain the generalization in a slight more principled manner, by restricting the type system. By not allowing the type $t$ for denotations, we prevented violations of the three original generalizations. Some questions still remain, however, since we had to stipulate that the operators required to apply a situation argument to a predicate were lexically linked to strong determiners and the $\beta$ operator. Also, the reason why a strong determiner applies its situation argument to its restrictive clause instead of its nuclear scope is left unexplained. The trade-off for these few stipulations, though, is a very simple system that explains all three original generalizations parsimoniously. 


\section{References}

Büring, D.: 2005, Binding Theory. Cambridge University Press.

Chierchia, G.: 1998, 'Reference to Kinds across Language', Natural Language Semantics 6, 339-405.

Cresswell, MJ: 1973, Logics and Languages. London: Methuen.

Cresswell, M.J.: 1990, Entities and indices. Kluwer Academic Publishers Boston.

Heim, I. and A. Kratzer: 1998, Semantics in Generative Grammar. Blackwell Publishers.

Jackendoff, R.: 1977, $X^{\prime}$ syntax. MIT Press Cambridge, Mass.

Keshet, Ezra: 2008, Good Intensions: Paving Two Roads to a Theory of the De re/De dicto Distinction, Doctoral Dissertation, MIT.

Kratzer, A.: 1991, 'Modality', Semantics: An International Handbook of Contemporary Research 639-650.

Kusumoto, K.: 2005, 'On the Quantification over Times in Natural Language', Nat. Lang. Sem. 13, 317-357.

Musan, R.: 1997, On the temporal interpretation of noun phrases. Garland.

Percus, O.: 2000, 'Constraints on Some Other Variables in Syntax', Natural Language Semantics 8, 173-229.

Schueler, D.: 2007, 'World Variable Binding and Beta Binding', Proceedings of NELS 38. 